MATEC Web of Conferences 11,03016 (2014)

DOI: $10.1051 /$ matecconf / 20141103016

(C) Owned by the authors, published by EDP Sciences, 2014

\title{
Assessment of metal pollution in the river of Martil emerging in the western Moroccan Mediterranean. Mobility and impact of human activities
}

M. RAISSOUNI ${ }^{1}$, Z. DJEBARY TAHIRI ${ }^{1}$, A. RAISSOUNI ${ }^{1}$,A. EL KADI ${ }^{1}$,A. EL ARRIM ${ }^{1}$ and H. ER-RAIOUI ${ }^{2}$

1. Laboratoire d'Océanologie, Environnement et Risques Naturels. Département des Sciences de la Terre ; Faculté des Sciences et Techniques. BP 416 Tanger. Maroc. Email : $\underline{\text { med.raissouni@gmail.com }}$

2. Laboratoire de Biologie Appliquée et Sciences de l’Environnement. Département des Sciences de la Terre. FST de Tanger. BP 416. Tanger. Maroc.

\begin{abstract}
This study is part of a monitoring of the evolution of variations in time and space contents of metallic elements of surface waters of the river of Martil between two sampling campaigns (May 2010 and May 2011). The comparison of the results of metal analyzes of the two campaigns show different variations of the contents of metallic elements. The magnesium content increased almost 10 times, which highlights the intensity of activities that caused this increase for one year only. The concentrations of nickel and lead fell below standards and indicate a water medium to high quality, as the concentrations of aluminum, zinc and copper, which have been reduced compared to the 2010 campaign and are still very low and do not pose a threat so large. The concentration of cadmium also decreased slightly but remains high above the standard set which is $5 \mathrm{mg} / 1$, which raises some concern about this element and its possible sources. For iron, its concentration was doubled, which is why it is a beginning of water contamination because the recorded values are between 1 and 2 , indicating an average water quality.
\end{abstract}

\section{Introduction}

La zone littorale qui est un écosystème fragile subit de différentes forces aux niveaux lithologiques, morphologiques et écologiques et par conséquent elle est soumise à des divers processus d'évolution. Sa position et son rôle socio-économique capital impose un aménagement et une protection efficaces.

L'objectif de notre étude est de suivre l'évolution de la qualité des eaux de surface de l'oued Martil, qui débouche dans la méditerranée occidentale marocaine, à partir de l'étude de leurs paramètres physicochimiques et leurs teneurs en quelques métaux lourds, ainsi de déterminer l'impact des rejets urbains et industriels sur l'environnement fluviatile. C'est une étude comparative de deux campagnes d'échantillonnage effectuées au mois de mai 2010 et au mois de mai 2011 respectivement.

\subsection{Localisation}

Le bassin versant de Martil draine une superficie de $1170 \mathrm{~km}^{2}$. Son cours d'eau principal traverse la ville de Tétouan avant de se déverser dans la Méditerranée au niveau du centre de la ville de Martil. Il est localisé dans la partie occidentale de la chaîne montagneuse du Rif. La zone d'étude s'étend sur une aire géographique caractérisée par un paysage essentiellement montagneux et elle est classée parmi les régions à climat de type méditerranéen humide.

\subsection{Pétrographie}

Le bassin versant de Martil est localisée dans la partie occidentale de la chaîne montagneuse du Rif. Ce dernier appartient à la terminaison occidentale de la chaîne alpine péri-méditerranéenne.

Les écailles et barres de la chaîne calcaire prennent place sur la bordure orientale du bassin en une bande

\section{Le Site D'étude}

This is an Open Access article distributed under the terms of the Creative Commons Attribution License 2.0, which permits unrestricted use, distribution, and reproduction in any medium, provided the original work is properly cited. 
continue de long de part et d'autre de la cluse de Tétouan.

\section{Matériels et Méthodes}

Le choix des points a été basé d'abord sur la notion amont - aval et sur la position des rejets principaux (figure 1).

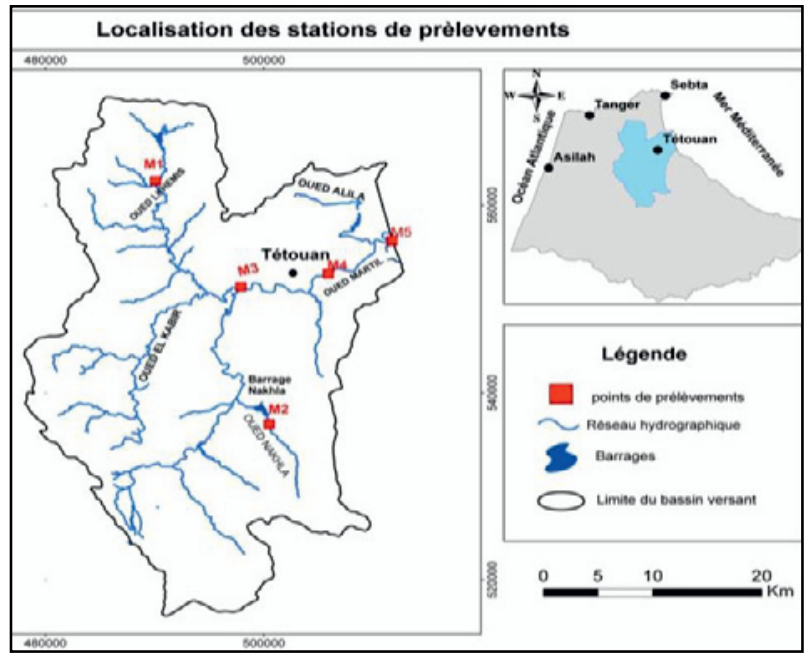

Fig.1. Localisation des points de prélèvement.

L'analyse des eaux de surface a porté sur la détermination des paramètres physicochimiques $(\mathrm{pH}$, température, conductivité, oxygène dissous, MES, DCO et DBO5) et sur le dosage de éléments métalliques cités précédemment par la technique ICP-AES.

\subsection{Paramètres physicochimiques}

\subsubsection{Paramètres in situ}

Les paramètres que nous avons mesurés sur place au point d'échantillonnage sont : le $\mathrm{pH}$, la température, la conductivité et l'oxygène dissous. Les 4 paramètres ont été mesurés à l'aide du même appareil multiparamètres et les résultats on été noté directement sur les fiches de prélèvements.

a. $\mathrm{pH}$

Le $\mathrm{pH}$ de l'eau résume la stabilité de l'équilibre établi entre les différentes formes de l'acide carbonique. Il est lié au système tampon développé par les carbonates et les bicarbonates (CO2-HCO3-CO3). Il dépend de la diffusion du gaz carbonique à partir de l'atmosphère, du bilan des métabolismes respiratoires et photosynthétiques. [2], ainsi que de l'origine des eaux, la nature géologique du milieu traversé, les rejets des eaux usées, etc. [3]. Des pH compris 5 et 9 permettent un développement à peu près normal de la faune et de la flore.

b. Conductivité

La conductivité électrique renseigne sur le degré de minéralisation globale des eaux superficielles. Des températures élevées agissent sur la conductivité électrique par action sur la mobilité des sels. [3]. Une conductivité élevée traduit soit des $\mathrm{pH}$ anormaux, soit le plus souvent une salinité élevée. [4].

c. Oxygène dissous

L'oxygène est l'un des facteurs fondamentaux de la vie. Il entre pour $21 \%$ dans la composition de l'air atmosphérique et représente $35 \%$ environ des gaz dissous dans l'eau à pression normale. [4].

La teneur en oxygène dissous dans les eaux naturelles sont déterminées principalement par:

- la respiration des organismes aquatiques ;

- l'oxydation et la dégradation des polluants ;

- et l'activité photosynthétique de la flore, les échanges avec l'atmosphère.

\section{d. Température}

Généralement, les variations de la température de l'eau sont tributaires des variations de la température atmosphérique ambiante et qui dépend elle aussi des variations saisonnières.

La température est un facteur important dans la vie d'un cours d'eau. L'élévation anormale de ce paramètre est due à un apport direct de la chaleur provenant des industries utilisant l'eau comme fluide réfrigérant. [4].

\subsubsection{Paramètres mesurés au laboratoire}

a. Matières en suspension (MES)

Les matières en suspension sont fonction de la nature des terrains traversés, de la saison, de la pluviométrie et des travaux des rejets.

En effet, tous les cours d'eau contiennent des matières en suspension de quelques milligrammes par litre ne posent pas de problèmes majeurs. Cependant, 
des teneurs élevées peuvent empêcher la pénétration de la lumière, diminuer l'oxygène dissous et limiter alors le développement de la vie aquatique en créant des déséquilibres entre les diverses espèces.

b. Demande Chimique en Oxygène (DCO)

La DCO correspond à la teneur de l'ensemble des matières organiques que celles-ci aient un caractère biodégradable ou non. Elle est exprimée par la quantité $\mathrm{d}^{\prime} \mathrm{O}_{2}$ fournie par le dichromate de potassium et nécessaire à l'oxydation des substances organiques présentes dans les eaux résiduaires.

c. Demande Biochimique en Oxygène (DBO)

La demande biochimique en oxygène est définie comme la quantité d'oxygène consommée par certaines matières présentes dans l'eau, pour assurer leur dégradation par voie biologique dans des conditions d'essai prédéfinies (après incubation durant 5 jours à $20^{\circ} \mathrm{C}$ et dans l'obscurité). Elle s'exprime en $\mathrm{mg}$ d'oxygène par litre d'eau (mg/l).

\subsection{Dosage des éléments métalliques}

\subsubsection{Méthode analytique}

L'étude a été faite sur onze éléments métalliques qui sont : l'aluminium ( $\mathrm{Al})$, le fer $(\mathrm{Fe})$, le magnésium $(\mathrm{Mg})$, le cadmium $(\mathrm{Cd})$, le plomb $(\mathrm{Pb})$, le Crome $(\mathrm{Cr})$, le cuivre $(\mathrm{Cu})$, , le nickel $(\mathrm{Ni})$, et le zinc $(\mathrm{Zn})$.

La méthode d'analyse effectuée est la torche à plasma par couplage à la spectrométrie d'émission optique ou l'ICP-AES « inductively coupled plasma-atomic emission spectrometry » qui été faite à l'Unité d'Appuie Technique à la Recherche Scientifique (UATRS) du Centre Nationale des Recherches Scientifiques et Techniques (CNRST) à Rabat.

\section{Résultats et discussions}

\subsection{Paramètres physicochimiques :}
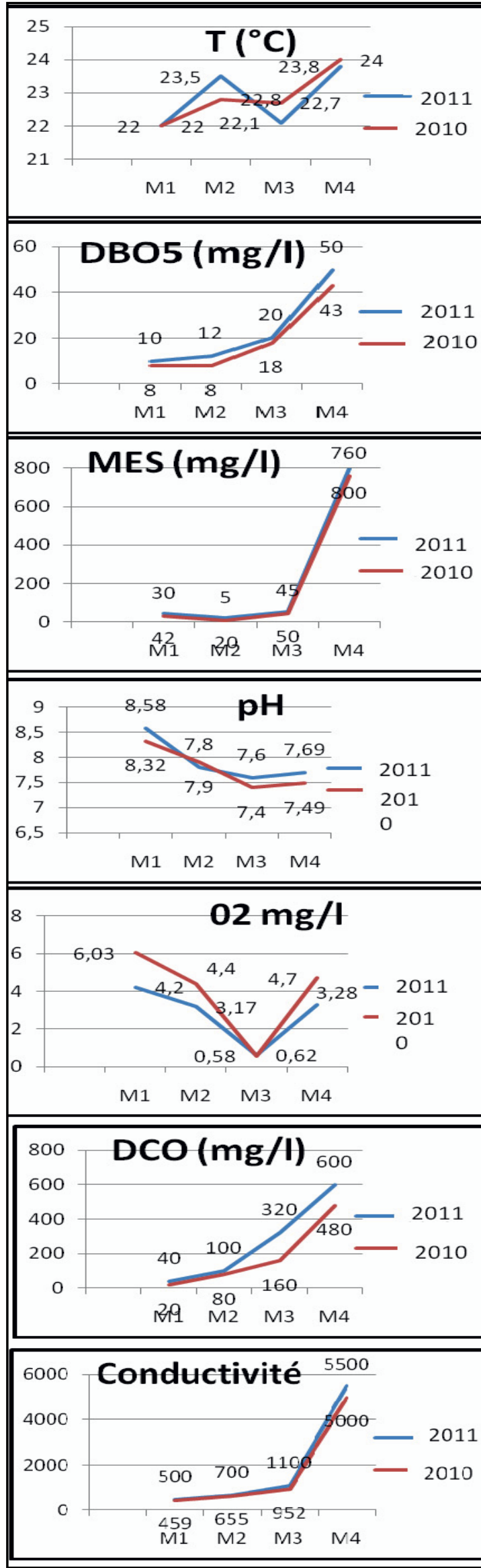

Fig. 2. Variation des paramètres physico-chimiques dans les différents points de prélèvement. 
Tableau 1: Tableau des résultats des paramètres physicochimiques des deux campagnes (2010 et 2011)

\begin{tabular}{|l|c|c|c|c|c|c|c|c|c|}
\hline $\begin{array}{c}\text { Points de } \\
\text { prélèvement }\end{array}$ & M1 & \multicolumn{2}{|c|}{ M2 } & \multicolumn{2}{|c|}{ M3 } & \multicolumn{2}{c|}{ M4 } & \multicolumn{2}{c|}{ M5 } \\
\hline Campagne & 2011 & 2011 & 2010 & 2011 & 2010 & 2011 & 2010 & 2011 & 2010 \\
\hline $\mathrm{O} 2 \mathrm{mg} / 1$ & 3,18 & 4,2 & 6,03 & 3,17 & 4,4 & 0,62 & 0,58 & 3,28 & 4,7 \\
\hline $\mathrm{pH}$ & 8,6 & 8,58 & 8,32 & 7,8 & 7,9 & 7,6 & 7,4 & 7,69 & 7,49 \\
\hline $\mathrm{T}^{\circ} \mathrm{C}$ & 23,4 & 22 & 22 & 23,5 & 22,8 & 22,1 & 22,7 & 23,8 & 24 \\
\hline $\mathrm{COND} \mu \mathrm{S} / \mathrm{cm}$ & 362 & 500 & 459 & 700 & 655 & 1100 & 952 & 5500 & 5000 \\
\hline $\mathrm{MES} \mathrm{mg} / 1$ & 15 & 42 & 30 & 20 & 5 & 50 & 45 & 800 & 760 \\
\hline $\mathrm{DCO} \mathrm{mg} / 1$ & 20 & 40 & 20 & 100 & 80 & 320 & 160 & 600 & 480 \\
\hline $\mathrm{DBO} 5 \mathrm{mg} / 1$ & 8 & 10 & 8 & 12 & 8 & 20 & 18 & 50 & 43 \\
\hline
\end{tabular}

\subsubsection{Paramètres in situ}

\section{a. L'oxygène dissous $(\mathrm{O} 2)$}

Pour les mesures de l'oxygène dissous, on voit qu'il ya une simple différence entre les valeurs, sauf dans le point M4 où il présente un minimum de $(0,62 \mathrm{mg} / \mathrm{l})$, situé après la zone industrielle, le maximum est au niveau du point $\mathrm{M} 2$, avec une valeur de $(4,20 \mathrm{mg} / \mathrm{l})$.

\section{b. Le potentiel d'hydrogène $(\mathrm{pH})$}

Pour le $\mathrm{pH}$, on note qu'il ya une diminution en allant de l'amont $(8,6)$ vers l'aval $(7,63)$, avec une légère diminution au niveau du point M4 $(7,6)$.

\section{c. La conductivité}

La conductivité au niveau d'oued varie entre la valeur maximale dans l'aval de $5500 \mu \mathrm{S} / \mathrm{cm}$ et 362 en amont, indiquant une charge ionique élevée.

\section{d. La température}

En général, la température est moyenne, elle est fonction de la température ambiante, elle varie de $22^{\circ} \mathrm{C}$ à $23,8^{\circ} \mathrm{C}$.

\subsubsection{Paramètres mesurés au laboratoire}

a. Les matières en suspension (MES)

Les teneurs en matières en suspension sont très faibles presque dans tous les points, sauf au niveau de l'aval avec une valeur élevée de $800 \mathrm{mg} / \mathrm{l}$ dépassant les normes établie. b. Demande Chimique en Oxygène (DCO)

La DCO, augmente de l'amont $(20 \mathrm{mg} / \mathrm{l})$ vers l'aval avec une valeur maximale de $600 \mathrm{mg} / \mathrm{l}$, qui est une valeur très élevée loin des normes établies ( $>80 \mathrm{mg} / \mathrm{l}$ ).

c. Demande Biochimique en Oxygène (DBO)

Généralement, la demande biochimique en oxygène dans le bassin augmente en allant de l'amont vers l'aval, jusqu'elle atteint une valeur de $50 \mathrm{mg} / \mathrm{l}$. Cette dernière dépasse les normes ( $>25 \mathrm{mg} / \mathrm{l})$.

\subsection{Elements métalliques :}

Pour le cadmium, les concentrations dépassent les normes établies, $(>5 \mu \mathrm{g} / \mathrm{l})$ presque dans tous les points. En effet, ce métal, provient des effluents industriels, des eaux usées domestiques et aussi des industries des alliages métalliques. Dans la nature, le Cd est l'un des rares éléments de la biosphère. Sa concentration moyenne dans la croûte terrestre est de l'ordre de 0,15 $\mu \mathrm{g} / \mathrm{g}$. [5].

Le magnésium, a des concentrations très élevées, dépassant beaucoup les normes établies (1 mg/l), le maximum est de 153,992 mg/l. Le nickel et le plomb en concentration dans la zone, affichent des valeurs qui ne dépassent pas les normes $(50 \mu \mathrm{g} / \mathrm{l})$, qualifiant la qualité des eaux de moyenne à bonne.

Les concentrations en zinc et en cuivre, sont très faibles, ils ne constituent pas de menace de manière général. Pour l'aluminium et le fer, ils affichent des concentrations qui augmentent en allant de l'amont du bassin vers l'aval, (1,534 mg/l) en aval pour le fer, et 0,573 $\mathrm{mg} / \mathrm{l}$ pour l'Al et comme l'étude des relations entre les éléments métalliques a fait apparaitre une corrélation positive entre ces deux éléments, on peut conclure qu'ils ont une origine commune, qui est les déchets d'utilisation d'aluminium et de fer et les activités industrielles présentes dans la zone, comme la construction (fenêtres, portes, gouttières), aussi les emballages alimentaires qui augmentent les concentrations de ces métaux. 


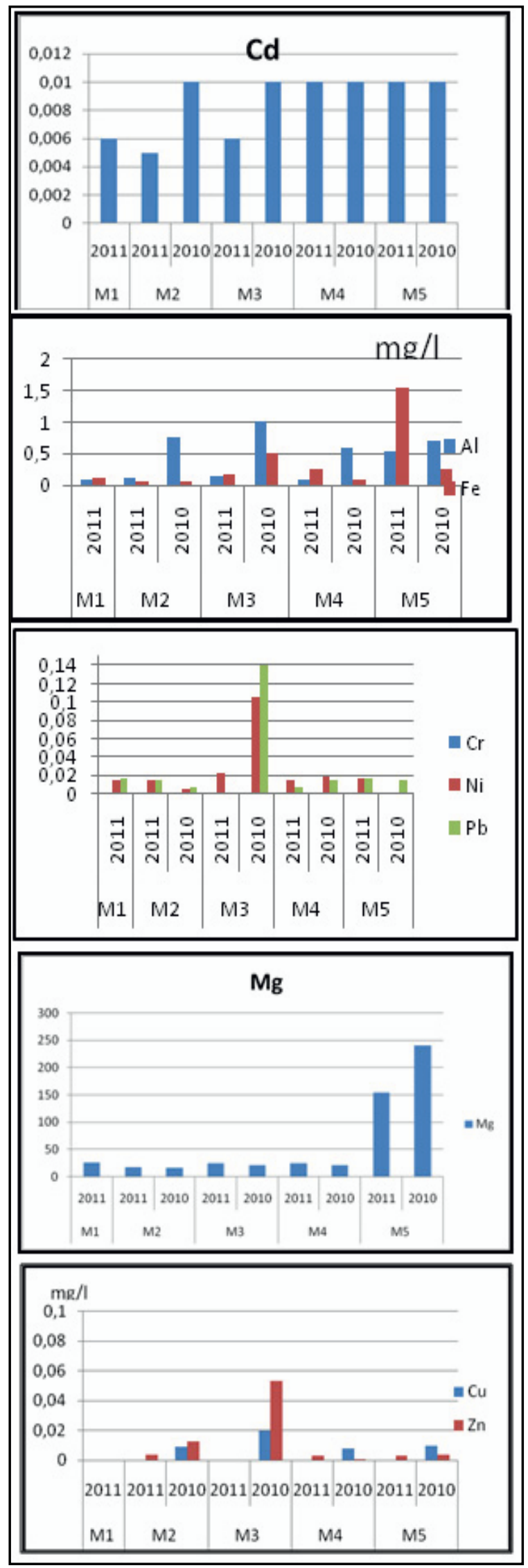

Fig. 3. Variation des concentrations des éléments métalliques dans les différents points de prélèvement.
Tableau 2: Résultats des analyses des éléments métalliques dans les eaux superficielles de l'oued Martil (mg/l).

\begin{tabular}{|l|c|c|c|c|c|c|c|c|c|}
\hline $\begin{array}{r}\text { Pts } \\
\text { de } \\
\text { prél }\end{array}$ & M1 & \multicolumn{2}{|c|}{ M2 } & \multicolumn{2}{c|}{ M3 } & \multicolumn{2}{c|}{ M4 } & \multicolumn{2}{c|}{ M5 } \\
\hline Ann & 2011 & 2011 & 2010 & 2011 & 2010 & 2011 & 2010 & 2011 & 2010 \\
\hline Al & 0,087 & 0,115 & 0,749 & 0,142 & 1,026 & 0,097 & 0,597 & 0,537 & 0,694 \\
\hline Fe & 0,113 & 0,064 & 0,074 & 0,163 & 0,516 & 0,268 & 0,083 & 1,534 & 0,25 \\
\hline Mg & 25,75 & 18,03 & 16,32 & 24,29 & 20,82 & 24,69 & 20,85 & 153,99 & 240,83 \\
\hline Cd & 0,006 & 0,005 & 0,01 & 0,006 & 0,01 & 0,01 & 0,01 & 0,01 & 0,01 \\
\hline Cr & $\leq 0,002$ & $\leq 0,002$ & 0,001 & $\leq 0,002$ & 0,002 & $\leq 0,002$ & 0,001 & $\leq 0,002$ & 0,002 \\
\hline Cu & $\leq 0,002 \leq 0,002$ & 0,009 & $\leq 0,002$ & 0,02 & $\leq 0,002$ & 0,008 & $\leq 0,002$ & 0,01 \\
\hline Zn & $\leq 0.002$ & 0,004 & 0,013 & $\leq 0.002$ & 0,053 & 0,003 & 0,001 & 0,003 & 0,004 \\
\hline $\mathrm{Ni}$ & 0,015 & 0,015 & 0,006 & 0,022 & 0,105 & 0,015 & 0,018 & 0,017 & 0,002 \\
\hline $\mathrm{Pb}$ & 0,017 & 0,015 & 0,008 & $\leq 0,004$ & 0,14 & 0,007 & 0,014 & 0,016 & 0,014 \\
\hline
\end{tabular}

\section{Conclusion}

Si les concentrations enregistrées n'incitent pas à des inquiétudes immédiates et ne peuvent être à l'origine de toxicité aiguë, il faudra souligner que le risque écotoxicologique réside dans le caractère cumulatif des métaux lourds qui interviennent dans des phénomènes de bioaccumulation et de bioamplification.

\section{Références bibliographiques}

1. MATEE -Ministère de l'Aménagement du Territoire, de l'Eau et de l'Environnement- (2005). Monographie de la province de Tétouan.

2. G.E. Hutchinson, (1957). Cold Spring Harbor Symposia on Quantitative Biology, 22 (2): 415-427. Retrieved 2007-07-24.

3. B. Dussart, (1966). Limnologie. In: L'étude des eaux continentales Gauthier-Villars, Collec. Géobiol. Ecol., Aménagement, Paris.

4. R. Bremond, B. Perrodon, (1979), Les paramètres de la qualité des eaux. Ministère de l'Environnement et du cadre de vie, 2ème édition, Neuilly sur Seine, Ministère de l'Environnement et du cadre de vie, Paris, $249 \mathrm{p}$. 5. L. LEVESQUE. (1980). Les rejets des eaux chaudes des centrales thermiques. I : Etudes sur sites des effets sur la vie aquatique. Association Française pour l'Etude des Eaux, Paris, 177p. 